\title{
Evaluación prospectiva de paratiroidectomías en el tratamiento del hiperparatiroidismo secundario*
}

\author{
Drs. JUAN ANTONIO PÉREZ P. ${ }^{1,2}$, MAURICIO GABRIELLI N. ${ }^{1,2}$, \\ MARIANNE BORN G. ${ }^{1,2}$, INT. PAULINA TRONCOSO G. ${ }^{3}$ \\ Instituto de Cirugía, Facultad de Medicina, Universidad Austral. \\ Subdepartamento de Cirugía, Hospital Base de Valdivia. \\ Interna Facultad de Medicina Universidad Austral. \\ Valdivia, Chile.
}

Abstract

\section{Parathyroidectomy for secondary hyperparathyroidism in chronic renal failure}

Background: Parathyroidectomy is indicated in patients with chronic renal failure and secondary hyperparathyroidism that is persistent and refractory to treatment. Aim: To assess the effects of parathyroidectomy in patients with chronic renal failure and symptomatic hyperparathyroidism. Patients and Methods: Prospective evaluation of 33 patients (20 females), aged between 23 and 78 years, with chronic renal failure and secondary hyperparathyroidism that were subjected to a total parathyroidectomy and autotrasplantation. Patients were followed for six months measuring parathormone (PTH), calcium and phosphorus. Results: In the postoperative period, one patient had a cervical hematoma that had to be operated. Mean serum PTH levels at the moment of hospital discharge and at six months, were 832 and 212 $\mathrm{pg} / \mathrm{ml}$ respectively. All patients that had bone pain experienced a notable reduction of this symptom on the morning after surgery. One patient had a relapse of her hyperparathyroidism. Conclusions: Parathyroidectomy is safe and effective for secondary hyperparathyroidism in patients with chronic renal failure.

Key words: Hyperparathyroidism, renal failure, parathormone.

\section{Resumen}

En los pacientes con insuficiencia renal crónica terminal con hiperparatiroidismo severo persistente y refractarios al tratamiento médico, está indicada la paratiroidectomía. El objetivo de este estudio es caracterizar una población y evaluar los efectos clínicos de la paratiroidectomía efectuada en pacientes con insuficiencia renal crónica terminal con sintomatología importante de hiperparatiroidismo y determinar la recurrencia de la enfermedad posterior a la cirugía. Se realizó un estudio prospectivo observacional de pacientes con hiperparatiroidismo, secundario a insuficiencia renal, que ingresaron al subdepartamento de cirugía del Hospital Base de Valdivia, entre abril de 1999 y noviembre de 2006 y que participaron voluntariamente en un protocolo preestablecido. La serie incluye a 33 pacientes, 20 mujeres y 13 hombres, con

*Recibido el 26 de Agosto de 2008 y aceptado para publicación el 16 de Septiembre de 2008.

Correspondencia: Dr. Juan A. Pérez P.

Arturo Günther 1664, Valdivia, Chile.

E-mail: jperez.dr@gmail.com 
promedio de edad de 43 años y extremos de 23 y 78 años, que fueron sometidos paratiroidectomía subtotal o total más autotrasplante. En el postoperatorio un enfermo presentó un hematoma cervical, que ameritó una reintervención quirúrgica, con evolución posterior satisfactoria. Los niveles de PTH al ingreso y a los 6 meses de operados los pacientes, fue en promedio de $832 \mathrm{pg} / \mathrm{ml}$ y $212 \mathrm{pg} / \mathrm{ml}$ respectivamente. Todos los pacientes que manifestaron dolor óseo como síntoma principal, advirtieron una notable disminución subjetiva del dolor a la mañana siguiente de la cirugía. Un paciente $(3 \%)$ evolucionó hacia la recidiva de su hiperfunción paratiroidea. Concluimos que la paratiroidectomía es un procedimiento seguro, que resulta efectivo para aliviar rápidamente la sintomatología que afecta a este tipo de pacientes y que el porcentaje de recidiva que hemos constatado en un seguimiento a seis meses postoperatorio es bajo.

Palabras clave: Hiperparatiroidismo secundario, paratiroidectomía, autotrasplante.

\section{Introducción}

El déficit mantenido de calcitriol secundario a la insuficiencia renal crónica (IRC) es la principal causa de hipertrofia paratiroídea y del aumento de los niveles de parathormona (PTH) ${ }^{1}$. En casos avanzados de insuficiencia renal, cuando el hiperparatiroidismo secundario es refractario al tratamiento médico se recurre a la paratiroidectomía.

En 1994, la European Dialysis and Transplantation Registry Report, reportó que el 1,3\% de los pacientes con IRC en hemodiálisis requirieron de una paratiroidectomía, debido principalmente al dolor óseo severo y al prurito que les provocaba el hiperparatiroidismo secundario ${ }^{2}$.

Para tratar esta condición se han propuesto tres tipos de cirugía. La primera, descrita en 1960, fue la paratiroidectomía subtotal es decir resecar todas las paratiroides pero conservando un remanente de tejido paratiroídeo; posteriormente se propuso la paratiroidectomía total la cual rápidamente perdió adeptos a causa de la osteomalacia ulterior con que evolucionaban los pacientes. No obstante, en los años siguientes se demostró que la osteomalacia no obedecía a la eliminación completa del tejido paratiroídeo, sino más bien a la acumulación preexistente de aluminio en el hueso, con lo cual la técnica recuperó su validez ${ }^{3}$.

Finalmente, en 1968 Alberyd reportó la primera experiencia de una paratiroidectomía total más autotrasplante de tejido paratiroídeo fresco ${ }^{4}$. Desde entonces, se ha considerado que la paratiroidectomía subtotal y la total más autotrasplante, son procedimientos que proporcionan resultados similares. En 1991 Rothmund $^{5}$, publicó un ensayo clínico en donde comparaba ambas técnicas, y concluyó que la paratiroidectomía total más autotrasplante, es mejor para pacientes con osteodistrofia renal siendo en la actualidad una técnica mundialmente aceptada.

El objetivo de este estudio es caracterizar una población y evaluar los efectos clínicos de la paratiroidectomía subtotal y total más autotransplante efectuada en pacientes con insuficiencia renal cró- nica terminal con sintomatología severa de hiperparatiroidismo y determinar la recurrencia de la enfermedad posterior a la cirugía.

\section{Material y Método}

Entre abril de 1999 y diciembre de 2006, en el subdepartamento de cirugía del Hospital Base Valdivia, se intervinieron quirúrgicamente 33 pacientes portadores de hiperparatiroidismo secundario a IRC. La cirugía consistió en una paratiroidectomía subtotal o total más autotrasplante de tejido paratiroideo alojado en un músculo esternocleidomastoídeo. Todas las cirugías fueron llevadas a cabo por el mismo cirujano.

Durante el período en estudio, los pacientes que ingresaban por hiperparatiroidismo secundario a IRC, sintomático, refractario a tratamiento médico, participaron voluntariamente en un protocolo que establecía las características demográficas de los pacientes (edad, sexo) y la sintomatología principal que los afectaba. A todos se les informó que participarían en un estudio prospectivo, observacional que pretendía principalmente caracterizar a la población en estudio y que no tendría ningún beneficio ni menoscabo en el tratamiento de su patología.

Previo a la cirugía los pacientes contaban con niveles séricos de PTH, Ca, P, Ca x P los que fueron repetidos al alta y a los 6 meses, a todos se les había realizado un cintigrama paratiroídeo.

Los enfermos fueron sometidos a diálisis al menos 24 horas previas a la cirugía.

La técnica quirúrgica, consistió en una cervicotomía transversa arciforme, sección del rafe de los músculos pretiroídeos y exploración de los territorios paratiroídeos. En todos los casos, al momento de resecar los nódulos paratiroideos se identificó el nervio laríngeo recurrente en ambos lados, con el fin de evitar su daño. El cirujano utilizó un optivisor con un aumento de $2 \mathrm{x}$ y cuando fue necesario ligar algún vaso sangrante se utilizó vicryl ${ }^{\mathrm{R}}$ $4 / 0$. Una vez realizada la paratiroidectomía total se obtuvo un trozo de una glándula extirpada, el que 
fue fraccionado e injertado en el espesor de las fibras de un esternocleidomastoideo quedando el sitio señalado con un clip metálico y, en el caso de la paratiroidectomía subtotal, se dejó una pequeña parte de una glándula in situ. En ningún paciente se dejó drenaje cervical.

Todas las glándulas extirpadas fueron enviadas a análisis histológico.

En relación al alivio de la sintomatología, dirigidamente a la mañana siguiente de la cirugía (todos los pacientes fueron operados a las 14:00 pm del día anterior), se consultó respecto a la cuantía del dolor esquelético que sentían comparativamente con el día anterior, respuesta que fue consignado utilizando la escala análoga del dolor de 1 a 10 .

El seguimiento fue prospectivo y observacional de al menos 6 meses, y llevado a cabo por el equipo de cirugía y nefrología del Hospital Base Valdivia, período en que se controló, y evaluó la remisión de la sintomatología, la recidiva de enfermedad y las posibles complicaciones.

\section{Resultados}

En el período en estudio, se operaron 33 pacientes por hiperparatiroidismo secundario a insuficiencia renal crónica terminal, de los cuales 20 fueron mujeres y 13 hombres. El promedio de edad fue de 43 años, con extremos de 23 y 78 años. La indicación quirúrgica en todos los pacientes fue la mala respuesta al tratamiento médico destinado a aliviar la sintomatología que los afectaba. En nuestra casuística las indicaciones principales que motivaron la cirugía fueron elevados niveles de PTH con o sin hipercalcemia, dolor óseo, debilidad muscular, fractura patológica y prurito (Tabla 1). Los niveles de PTH preoperatorios tuvieron una media de $832 \mathrm{pg} / \mathrm{ml}$.

El cintigrama paratiroídeo fue practicado en todos los paciente y evidenció 1 ó 2 zonas tejido paratiroídeo hiperfuncionante habitualmente en relación a los polos inferiores de la glándula tiroides.

Tabla 1. Causas que motivaron la cirugía de los pacientes

\begin{tabular}{lcr}
\hline Síntoma o signo & n pacientes & $\%$ \\
\hline Dolor óseo & 15 & 45 \\
Debilidad muscular & 7 & 21 \\
Fractura patológica & 4 & 12 \\
Prurito & 2 & 6 \\
Hipercalcemia severa & 5 & 15 \\
\hline
\end{tabular}

De los 33 pacientes operados, a 21 se le realizó una paratiroidectomía total más autotrasplante y a los 12 restantes una paratiroidectomía parcial. La decisión de realizar una paratiroidectomía total o subtotal se basó, principalmente en los hallazgos encontrados en la exploración cervical en relación al número y tamaño de las paratiroides ubicadas, y además en los hallazgos preoperatorios de la cintigrafía paratiroídea. De los 21 pacientes con paratiroidectomía total más autoinjerto en uno de ellos se encontró 5 glándulas paratiroides. En los pacientes en que se realizó una paratiroidectomía parcial, en promedio se extrajeron 2,54 glándulas. En una paciente se encontró un cáncer papilar de tiroides incidental realizándose la correspondiente tiroidectomía.

El calcio basal al ingreso tuvo una media de 10,51 $\mathrm{mg} / \mathrm{dl} \pm 1,32$, al alta de $8,55 \mathrm{mg} / \mathrm{dl} \pm 0,83$ y a los 6 meses de 9,23 mg/dl $\pm 1,32$ (Figura 1). En relación al fósforo en los mismos períodos las medias fueron: $5,02 \pm 0,33,4,34 \pm 1,37,4,48 \pm 1,27$, respectivamente (Figura 2). El producto calcio-fósforo, se comportó como se muestra en el Figura 3.

Durante el postoperatorio, los pacientes permanecieron con terapia de sustitución de calcio ev (8 ampollas de gluconato de calcio en $500 \mathrm{ml}$ de solución físiológica), un promedio de 5 días, con un mínimo de 1 día y un máximo de 20 días. Cabe mencionar la asociación de calcio oral o calcitriol según los requerimientos de cada paciente y a sus niveles de fosfemia.

Los pacientes se mantuvieron hospitalizados un promedio de 11 días, con un mínimo de 3 días y un máximo de 54 días. El paciente con más larga estadía presentó una neumonía que requirió ventilación mecánica.

En la serie no hubo mortalidad. Un paciente presentó un hematoma cervical, por lo que tuvo que ser reintervenido, con evolución posterior satisfactoria. Dos pacientes presentaron disfonía transito-

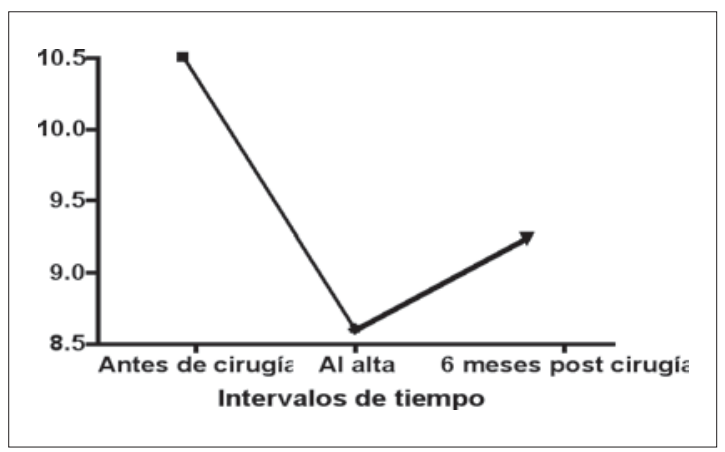

Figura 1. Niveles de calcio antes de la cirugía, al alta y a los 6 meses. 


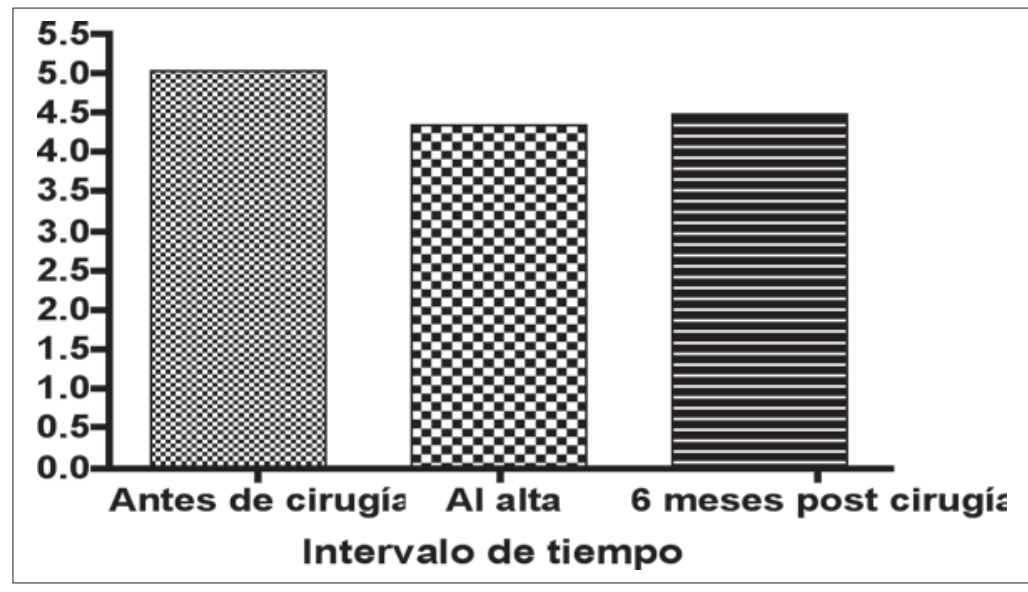

Figura 2. Niveles de fósforo antes de la cirugía, al alta y a los 6 meses.

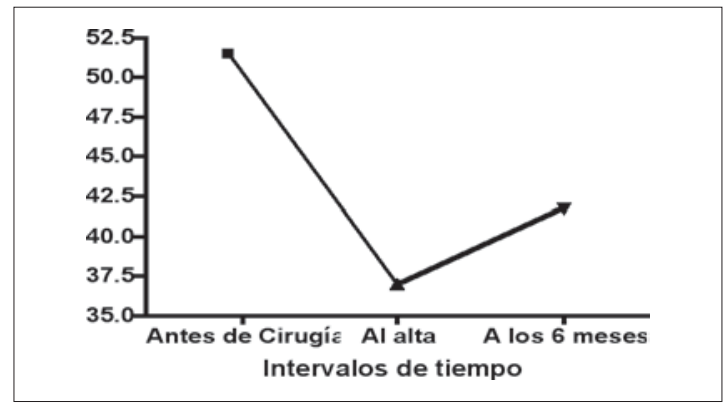

Figura 3. Producto calcio-fósforo antes de la cirugía, al alta y a los 6 meses.

ria posterior a la operación que se corrigió espontáneamente antes de los 2 meses del postoperatorio.

Referente al alivio de la sintomatología, la totalidad de los pacientes que sentían dolor óseo como síntoma principal (15 pacientes) presentaron una considerable disminución su malestar a la mañana siguiente de la cirugía, que en promedio fue de 4 puntos en la escala análoga de 1 a 10 . A los dos pacientes en que la sintomatología principal fue el prurito, este desapareció en su totalidad al primer día post cirugía. Los niveles de PTH al ingreso, al alta y a los 6 meses de operados los pacientes, fue en promedio de $832 \mathrm{pg} / \mathrm{ml}, 84 \mathrm{pg} / \mathrm{ml}$ y $212 \mathrm{pg} / \mathrm{ml}$ respectivamente.

Todas las glándulas fueron enviadas a biopsia diferida comprobándose su índole de paratiroides hiperplásicas. Se constató un cáncer papilar de tiroides y no se encontró ningún carcinoma paratiroídeo.

El total de pacientes fue controlado a los 6 meses y sólo se ha pesquisado una recidiva que se manifestó con reaparición de los dolores óseos y niveles altos de PTH.

\section{Discusión}

Desde que Massry y Goldstein ${ }^{6}$, reportaron los efectos deletéreos del HPT en el paciente urémico, se estableció que esta condición no sólo afectaba al sistema esquelético sino también a otros órganos especialmente cuando se asociaba con hiperfosfatemia ${ }^{7}$. Concretamente, el riesgo de mortalidad por causa cardiovascular en la población en diálisis supera todos los otros riesgos de muerte y amerita que la prevención de HPT sea obligatoria.

El hiperparatiroidismo secundario es una clásica manifestación de la IRC que en la mayoría de los casos puede ser tratado médicamente con buenos resultados. Cuando esto no ocurre la paratiroidectomía parcial o total más autotrasplante es una excelente alternativa terapéutica.

Las manifestaciones clínicas que provocan los trastornos causados por el HPT incluyen dolores óseos, debilidad muscular, deformidades esqueléticas (tórax en tonel, genu valgo), desinserciones y rupturas tendinosas, calcifilaxis, fracturas espontáneas, calcificaciones extra esqueléticas (secundarias a producto $\mathrm{Ca} \times \mathrm{P}$ elevado) y prurito entre otras.

La indicación de paratiroidectomía es pertinente en los pacientes con IRC terminal con hiperparatiroidismo severo refractario, con niveles persistentemente elevados de PTH intacta sobre $800 \mathrm{pg} / \mathrm{ml}$ sin respuesta a tratamiento médico ${ }^{8}$, asociado a hipercalcemia severa y calcificaciones extra esqueléticas progresivas o calcifilaxis que generalmente están en concomitancia con hiperfosfatemia refractaria a quelantes del fósforo. Si a lo anterior se agrega detección de paratiroides aumentadas de tamaño certificado por imágenes y enfermedad ósea hiperparatiroídea debilitante y progresiva, definida por evaluación radiológica, la paratiroidectomía se plantea como imperativa. 
La tendencia actual es evitar o minimizar las complicaciones cardiovasculares y con ese propósito se ha propuesto modificar las indicaciones quirúrgicas y es así como pacientes con valores persistentes de PTH $>500 \mathrm{pq} / \mathrm{ml}$, hipercalcemia $>10,2$ $\mathrm{mg} / \mathrm{dl}$ e hiperfosfatemia de $6 \mathrm{mg} / \mathrm{dl}$ que no pueden ser controladas mediante tratamiento médico sumado a paratiroides aumentadas de tamaño en la ultrasonografía, tendrían indicación quirúrgica, más aún si arrastran trastornos esqueléticos severos ${ }^{9}$.

Es oportuno recalcar que en los últimos años se ha demostrado que la paratiroidectomía reduce los eventos cardiovasculares y disminuye la mortalidad en los pacientes con hiperparatiroidismo secundario renal ${ }^{10}$.

La mejor cirugía para el manejo del hiperparatiroidismo secundario está aún en discusión ${ }^{11}$. En teoría, la paratiroidectomía total más autotrasplante tendría mayores ventajas comparativas, puesto que la recurrencia es más fácil de pesquisar y se puede solucionar con una intervención menor destinada a resecar el tejido paratiroídeo del lecho muscular donde fue trasplantado ${ }^{12}$. Dado que los sitios habituales en donde se realiza el trasplante son músculos de antebrazos o cuello, sin duda la reexploración cervical conlleva mayor morbilidad.

Sin embargo, algunos autores preconizan la paratiroidectomía subtotal, pues afirman que en caso de una recurrencia, ésta sólo podría ocurrir en el cuello o en el mediastino, y no en tres lugares como en la paratiroidectomía total, si se considera además, el sitio del autotrasplante. Casanova y $\operatorname{cols}^{13}$, describen una nueva e interesante forma de solucionar este problema, realizando isquemia completa en la extremidad en donde se realizó el autotrasplante; esto generaría un rápido cambio en la concentración de PTH si es que el hiperparatiroidismo es dependiente de la zona de autotrasplante. Pero en caso, en que el autotrasplante se realice en el músculo esternocleidomastoídeo, como acontece en nuestra casuística, este método carece de aplicación.

Cabe mencionar que en caso de una recidiva en el tejido autotrasplantado, la extirpación puede que resulte dificultosa puesto que es frecuente encontrar una importante infiltración de éste sobre los planos musculares, más aún si hay hiperplasia nodular.

Si bien los efectos a corto plazo obtenidos con ambas técnicas quirúrgicas se reportan como excelentes, en seguimientos prolongados una adecuada función paratiroidea se lograría en sólo en un tercio de los pacientes, la otras dos tercera partes desarrollarían hiperparatiroidismo recurrente o quedarían hipoparatiroídeos ${ }^{14}$. Para los pacientes que desarrollan un hipoparatiroidismo existe el recurso de los bancos para la criopreservación de paratiroides antólogas, siendo el éxito promedio del autoinjerto criopreservado de alrededor de un $60 \%$, logrando una función paratiroídea completa en un $40 \%$ de los casos. La duración de la criopreservación es un predictor clave de fracaso del injerto, y en torno a los 22 meses de criopreservación la función de un eventual injerto es prácticamente nula ${ }^{15}$.

Es importante mencionar además, el rol que cumple la paratiroidectomía total más autoinjerto en pacientes con HPT secundario sintomático que van a ser sometidos a un trasplante renal, puesto que puede favorecerse notoriamente los resultados de este último al producirse un significativo aumento de la densidad mineral ósea ${ }^{16}$.

Respecto a los resultados de nuestra experiencia, podemos afirmar que en cirujanos experimentados la paratiroidectomía total o subtotal es un procedimiento seguro, de muy baja morbilidad y que resulta muy efectivo para aliviar rápidamente la sintomatología que afecta a los pacientes. El porcentaje de recidiva es de un $3 \%$ a seis meses de seguimiento, sin embargo es predecible que en controles a mayor plazo, de acuerdo a la evolución natural de esta entidad clínica, éste se modificará y será más significativo.

\section{Referencias}

1. Mark SJ. Hyperparathyroid and hypoparathyroid disorders. N Engl J Med 2000; 343: 1863-1875.

2. Valderrabano F, Berthoux FC, Jones EH, Mehls O. Report on management of renal failure in Europe, XXV, 1994 end-stage renal disease and dialysis report. The EDTA-ERA registry. European Dialysis and Transplant-European Renal Association. Nephrol Dial Transplant 1996; 11: 2-21.

3. Richards ML, Wormuth J, Bingener J, Sirinek K. Parathyroidectomy in secondary hyperparathyroidism: Is there an optimal operative management? Surgery 2006; 139: 174-180.

4. Alveryd A. Parathyroid glands in thyroid surgery. I. Anatomy of parathyroid glands. II. Postoperative hypoparathyroidism, identification and autotransplantation of parathyroid glands. Acta Chir Scand Suppl 1968; 389: 1-120.

5. Rothmund M, Wagner PK, Schark C. Subtotal parathyroidectomy versus total parathyroidectomy and autotransplantation in secondary hyperparathyroidism: a randomized trial. World J Surg 1991;15: 745-750.

6. Massry SG, Goldstein DA. The search for uremic toxin (s) ,x", x" = PTH. Clin Nephrol 1979; 11: 181-189.

7. Bro S, Olgaard K. Effects of excess PTH on nonclassical 
target organs. Am J Kidney Dis 1997; 30: 606-620.

8. National Kidney Foundation. K/DOQI clinical practice guidelines for bone metabolism and disease in chronic kidney disease. Am J Kidney Dis 2003; 42: S1-202.

9. Tominaga Y, Matsuoka S, Sato T. Surgical indications and procedures of parathyroidectomy in patients with chronic kidney disease. Ther Apher Dial 2005; 9: 44-47.

10. Costa-Hong V, Jorgetti V, Gowdak LH, Moyses RM, Krieger EM, De Lima JJ. Parathyroidectomy reduces cardiovascular events and mortality in renal hyperparathyroidism. Surgery 2007; 142: 699-703.

11. Nichol PF, Mack E, Bianco J, Hayman A, Starling J, Chen H. Radioguided parathyroidectomy in patients with secondary and tertiary hyperparathyroidism. Surgery 2003; 134: 713-717.

12. Zaraca F, Mazzaferro S, Catarci M, Saputelli A, Alo $\mathrm{P}$, Carboni M. Prospective evaluation of total parathyroidectomy and autotransplantation for the treatment of secondary hyperparathyroidism. Arch
Surg 1999; 134: 68-72.

13. Casanova D, Sarfati E, De Francisco A, Amado J A, Arias M, Dubost C. Secondary hyperparathyroidism: diagnosis of site of recurrence. World J Surg 1991; 15 : 546-550.

14. Gagné ER, Ureña $P$, Leite-Silva $S$, Zingraff J, Chevalier A, Sarfati E, et al. Short- and long-term efficacy of total parathyroidectomy with immediate autografting compared with subtotal parathyroidectomy in hemodialysis patients. J Am Soc Nephrol 1992; 3: 1008-1017.

15. Cohen M, Dilley W, Wells S, Moley J, Doherty G, Sicard G, et al. Long-term functionality of cryopreserved parathyroid autografts: A 13-year prospective analysis. Surgery 2005; 138: 1033-1041.

16. Chou FF, Hsieh KC, Chen YT, Lee CT. Parathyroidectomy followed by kidney transplantation can improve bone mineral density in patients with secondary hyperparathyroidism. Transplantation 2008; 27: 554-557. 